\title{
Chapter 2 \\ Screening Protocols for Heat Tolerance in Rice at the Seedling and Reproductive Stages
}

\subsection{Background Analysis}

The optimum temperature for rice germination is between 28 and $30{ }^{\circ} \mathrm{C}$. High temperature affects almost all growth stages of rice from germination to ripening (Shah et al. 2011). The threshold temperature at the seedling stage has been identified as $35^{\circ} \mathrm{C}$; the main symptom of heat stress is poor growth (Yoshida 1981).

Prasanth et al. (2012) tested heat stress in different stages of plant and used 28 genotypes including three mutant lines. These authors noted genotype-specific response with regards to heat stress tolerance in the three different stage analysed: germination, seedling and early vegetative stage. They added that there are fewer reports on the effect of high temperature on germination and vegetative stage of rice seedlings compared to the reproductive stage.

Plant reproductive processes are complex and sensitive to environmental changes, including high temperatures, which ultimately affect fertilization and post-fertilization processes leading to decreased yields. Flowering is one of the most susceptible stages in the life cycle of rice, and rice spikelets at anthesis exposed to more than $35^{\circ} \mathrm{C}$ for 4-5 days induces sterility, with no seed produced (Satake and Yoshida 1978). Temperatures above $35{ }^{\circ} \mathrm{C}$ at flowering causes failure of anther dehiscence, and thus less pollen, resulting in incomplete fertilization in rice (Jagadish et al. 2007; Prasad et al. 2006; Satake and Yoshida 1978). Weerakoon et al. (2008) using a combination of high temperatures $\left(32-36{ }^{\circ} \mathrm{C}\right)$ with low $(60 \%)$ and high $(85 \%)$ relative humidity recorded high spikelet sterility. Flowering (meiosis, anthesis and fertilization) is considered to be one of the most sensitive stages for temperature stress in rice. The threshold temperature for success in flowering in rice is $33{ }^{\circ} \mathrm{C}$ (Jagadish et al. 2007). Therefore, the fertility of spikelets at high temperature can be used as a screening tool for high temperature tolerance (Satake and

The original version of this chapter was revised. A correction to this chapter is available at https://doi.org/10.1007/978-3-319-77338-4_5. 
Yoshida 1978). Some cultivars that are not so sensitive to higher temperatures, e.g. cv. N22, can be used in breeding programmes as controls in screening tests. Besides spikelet fertility occurring during the flowering stage, a phenotypic marker for male gametogenesis has been reported as a screening tool for high temperature stress in rice (Jagadish et al. 2014). Authors identified a distance of 8-9 $\mathrm{cm}$ between the collar of the last fully opened leaf and the flag leaf collar as the environmentally stable phenotypic marker to assess heat stress $\left(38{ }^{\circ} \mathrm{C}\right)$ sensitivity at the micro sporogenesis stage leading to high spikelet sterility in rice.

Wei-hun et al. (2012) applied heat stress at 15 day-old seedling stage $43{ }^{\circ} \mathrm{C} / 30{ }^{\circ} \mathrm{C}$ (day/night and $14 \mathrm{~h} / 10 \mathrm{~h}$ ), $75 \%$ relative humidity for 7 days and observed that plant height, root length, shoot and root biomass were dramatically reduced, with heat tolerant genotypes being relatively less affected than sensitive ones. In this sense, we have included seedlings as a target for heat tolerance screening, to enable selection at the seedling stage by plant breeders. This method, as described in Sect. 2.2, is based on growing rice seedlings in glasshouse conditions using hydroponics or pots and exposing them to heat stress in a growth chamber. The seedling test is simple and rapid (4-6 days) and allows the simultaneous screening of several hundred seedlings at once depending on the size of available infrastructure. A list of equipment required for hydroponics hardware and stock solutions is given. Heat stress treatment was applied after 15 days of seedling transplantation to hydroponics or pots. Germplasm classifications for sensitive, intermediate and tolerant are also provided. Visual symptoms of heat stress include: reduced leaf area, yellowing of leaves, leaf tip burning, and entire leaf burning and leaf death. Assessment of seedlings was done according to a heat tolerance index, which is calculated for seedling biomass data using shoot, root, whole seedlings weight (fresh and dry), root, shoot length and seedling height. The test can be adapted to screen $\mathrm{M}_{2}$ individuals as well as $\mathrm{M}_{3}$ families from mutant populations (and other large breeding populations). A stress tolerance index (STI, Fernandez 1992) was used to identify/validate genotypes producing high yield under both stress and non-stress conditions; Blum (1988) stated that selection based on STI can result in genotypes with higher stress tolerance and yield potential to be selected.

Tenorio et al. (2013) and Rang et al. (2011) developed a phenotyping protocol for use in plant breeding in which a temperature treatment of $38{ }^{\circ} \mathrm{C}$ is used at the flowering stage. Our second protocol presented here is to screen for heat tolerance in rice at the flowering stage modified from Tenorio et al. (2013) and Rang et al. (2011). Flowering is most heat sensitive stage that can result in high sterility and yield loss in rice. As explained in Sect. 2.3, glasshouse grown plants are moved to a controlled environmental from the first day of anthesis for heat stress treatments, at $35.0-39.0{ }^{\circ} \mathrm{C} / 28{ }^{\circ} \mathrm{C}$ for $6-4 / 18-20 \mathrm{~h}$ with relative humidity $80 \%$ for $6-4$ days. After heat treatment plants may be rescued and returned to glasshouse under optimum temperature conditions to produce seed and for further evaluation. Spikelet fertility (seed set) is measured as an important predictive parameter for yield (Prasad et al. 2006; Rang et al. 2011). In our protocol also we used spikelet fertility at maturity to assess the heat tolerance of the tested genotypes. 


\subsection{Screening Protocol for Heat Tolerance in Rice at the Seedling Stage}

\subsubsection{Plant Materials}

Experiments were performed using controlled environment facilitates, test plant materials were compared to standard genotypes of known heat stress tolerance. Rice controls used at the PBGL (Austria), India, UR Tanzania and Pakistan are:

N22: a heat tolerant mutant, India origin

IR64: an intermediate heat tolerant genotype, from IRRI, Philippines

IR52: a heat susceptible genotype, from IRRI, Philippines

WAB 56-104: a heat susceptible genotype, Nigeria origin

The heat tolerance of the above standards in hydroponics and pots has been correlated with field performance, see Sect. 3.3. These standard materials can be requested free of charge from IRRI under a Standard Material Transfer Agreement. It is advisable to include local cultivars or breeding lines of known heat stress tolerance in tests.

\subsubsection{Screening for Heat Tolerance Using Hydroponics}

The equipment for hydroponics, the preparation of nutrient stock solutions and setting up of the hydroponics system are given in Bado et al. (2016).

\subsubsection{Seedling Establishment in Hydroponics}

The seedling establishment was conducted in a glasshouse set up for rice: $28 / 20{ }^{\circ} \mathrm{C}$ and $70 \%$ humidity in $16 \mathrm{~h}$ photoperiod. Test tanks were filled with distilled water until the water level is about $1 \mathrm{~mm}$ above the mesh of the platform. Dry seed were then placed into the wet compartments of the seed support platform. For $\mathbf{M}_{2}$ screening 20-40 seeds from one panicle is placed into one compartment $(6 \times 7 \mathrm{~cm})$ (Fig. 2.1a). For $\mathrm{M}_{3}$ and advanced lines/cultivars 3-5 seeds were placed into each $2 \mathrm{~cm}$ diameter compartment. Individual lines may be replicated within and among tanks (Fig. 2.1b). The test platforms were then covered with a lid for 5-6 days to promote germination in the dark. On day 3, the seed should show signs of germination (emergence of root and shoot) and the water should be replaced with half-strength Yoshida solution as vigorous seedlings require nutrients. Germinated seeds were allowed to grow for 6 days. The seedlings for subsequent heat stress treatment should be healthy, therefore odd-looking or diseased seedlings should be removed at this stage. If seed samples are not clean and rotting occurs during germination, these must also be removed. If symptoms are widespread, a new batch of seedlings and fresh hydroponics materials should be prepared. 

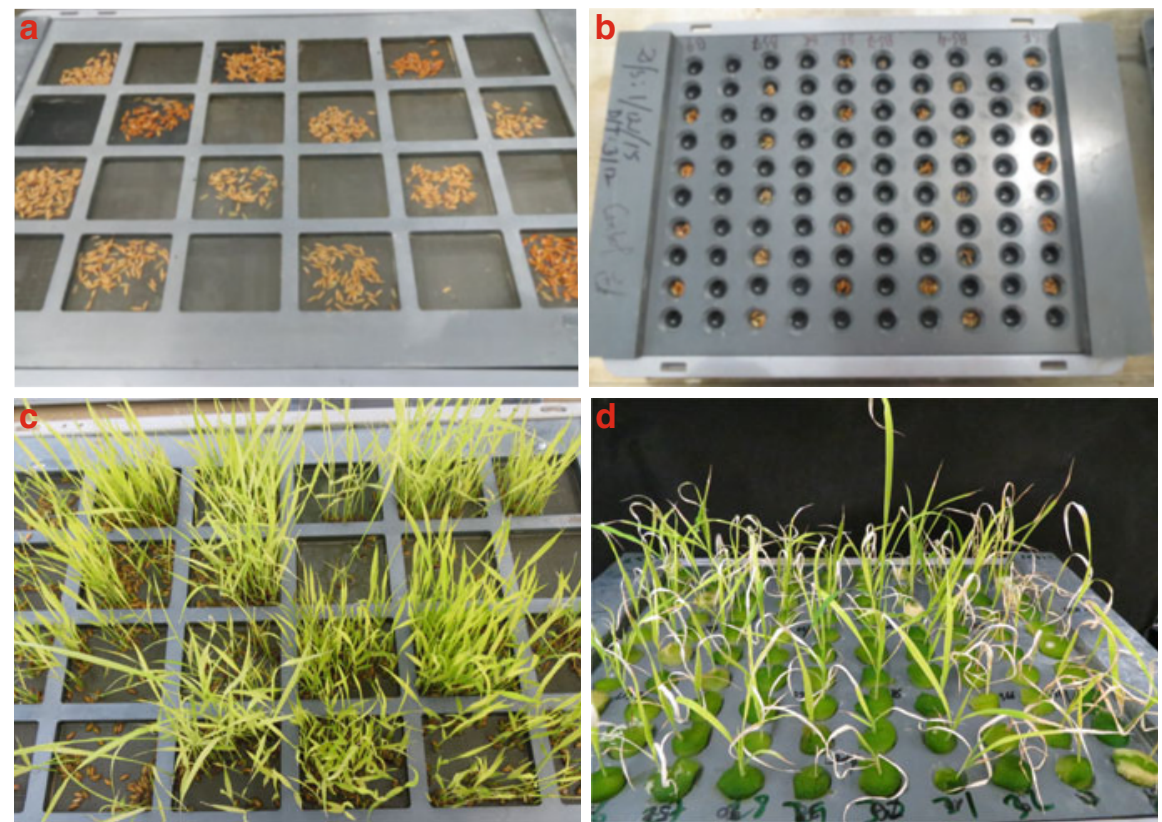

Fig. 2.1 Germination and seedling establishment for heat stress treatment in hydroponics. (a) Seed support platform with rice seeds ready for germination, each compartment contains seed from one panicle per $\mathrm{M}_{2}$ plant. (b) $\mathrm{M}_{3} / \mathrm{M}_{4}$ advanced line seed support platform showing rice seeds ready for germination, each compartment contains 3-5 seeds from each line. After germination reduced to one seedling for each compartment (c) Rice seedlings are ready for heat application. (d) After heat treatment, rice seedlings showing various degrees of heat injury, some rows all died (last two rows), some could show tolerance with less damage. (Pictures from PBGL, Joint FAO/IAEA Division)

\subsubsection{Care Required During Plant Growth in Hydroponics}

Due to evaporation from tanks and transpiration from plants there will be a gradual decrease in solution volumes over time. Every 2 days the volume needs to be brought back to the level of full capacity (touching the netting in the platform compartments) and the $\mathrm{pH}$ adjusted to 5 . If algal contamination is found, the nutrient solution should be completely replaced. Solutions can be changed by lifting off the platforms and placing them temporarily over empty tanks and pouring the hydroponics solutions back into a drum where the bulked solution can be $\mathrm{pH}$ adjusted for the whole experiment in one step. Once adjusted the solution is re-distributed into the test tanks and the seedling platforms returned. These operations also act to aerate the hydroponics solution. Alternatively, the $\mathrm{pH}$ can be adjusted on an individual tank basis and more working solution may be added to make up the volume in each tank. 


\subsubsection{Screening for Heat Tolerance in Soil Using Pots}

No special equipment is needed for screening with soil in pots and/or trays. Plastic pots, trays or tanks are filled with a clay soil which has a good water retention capacity and organic matter content. Pre-germinated, 6 day-old young healthy seedlings are transferred to the plastic pots, trays or tanks as given in Fig. 2.2a-c. Also as shown in Fig. 2.4d, in case germination problems, an excess (110-120) of seeds of each line are sown directly into the tray to have 100 seedlings in a tray 1 week after sowing.

Germinated 6 day-old seedlings were transferred to pots or trays to grown in a glasshouse set up for rice: $28 / 20^{\circ} \mathrm{C}$ day/night temperatures with $70 \%$ humidity and $16 \mathrm{~h}$ period. The glasshouse should be disease free and well-lit by natural or artificial lighting. Standard rice management practices are applied to pots with respect to soil, fertilizer and watering until the application of heat stress.


Fig. 2.2 Germination and seedling establishment for heat stress in trays. (a) Pre-germinated rice seedlings in petri dishes with filter paper. (b) Young seedlings transplanted into trays. (c) 15 days after transplantation seedlings are ready for heat treatment (d) $45{ }^{\circ} \mathrm{C}$ heat application in growth chamber (Pictures are from Sokoine University, URT) 


\subsubsection{Heat Treatment and Recovery of Seedlings}

Fifteen days after transplantation, seedlings were transferred to the growth chamber to grow under tightly controlled temperature, light and relative humidity conditions. Growth chambers were adjusted to $45^{\circ} \mathrm{C} / 28^{\circ} \mathrm{C}$ (day/night) $6 / 18 \mathrm{~h}$ with $80 \%$ relative humidity. The temperature treatment was imposed for 4-6 days; depending on seedling tolerance to heat stress application (some of them already died after 4 days). For all genotypes including control cultivars, 50/100 seedlings were exposed to heat stress and same amount of seedlings are grown in glasshouse conditions without stress as controls (Figs. 2.3 and 2.4).
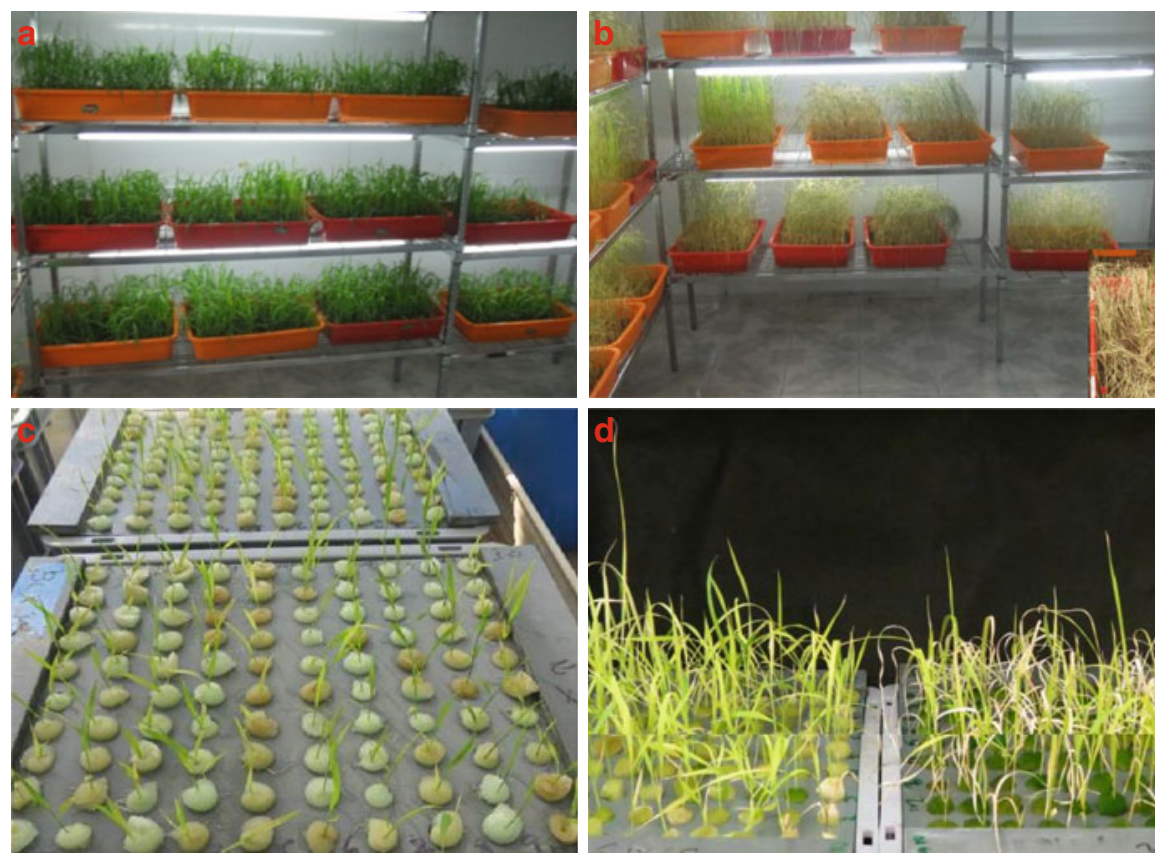

Fig. 2.3 Heat treatment to seedlings in trays/hydroponics, each tray contains 100 seedlings in control and stress conditions (a and c) Control seedlings no heat stress applied, (b) After heat stress treatment, heat stress effects on seedlings most of the genotypes already dead, one to two lines alive seems tolerant to heat stress (d) Seedlings in hydroponics ready for heat stress application, each tank contains 100 seedlings. Right side: hydroponically grown seedlings showing various degrees of heat injury, left side: control plants showing normal, good growth and health (Pictures from PBGL, Joint FAO/IAEA Division b and Sokoine University in URT) 

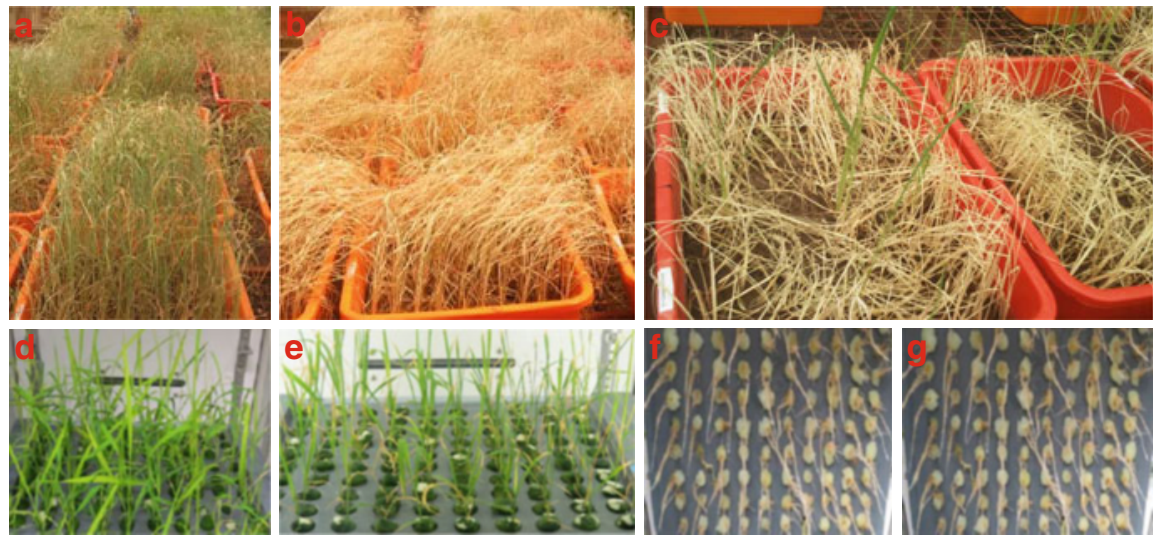

Fig. 2.4 Effects of heat stress application on seedlings. (a) After heat stress application some seedlings have survived and some have not. (b) All seedlings have died these lines are very sensitive to heat. (c) Only one to two seedling has survived and can be selected for multiplication. (d) Control (non-heat stress) healthy seedlings in hydroponics. (e) Heat stressed seedlings in hydroponics with injury to leaves some of them bad injured non tolerant to heat. (f) Control (non-heat applied) seedlings roots without damage. (g) After heat stress application, damage can be seen on roots of rice seedlings, bad injured leaves seedlings also have bad injured roots (Pictures from PBGL, Joint FAO/IAEA Division and Sokoine University, URT)

\subsubsection{Assessment of Heat Tolerance at the Seedling Stage}

Heat application to rice seedlings is illustrated in Fig. 2.5; visual symptoms of heat stress are: leaf yellowing, reduced leaf area, leaf tip burning, and entire leaf burning and leaf death. The biomass of seedlings is recorded as given Table 2.1 using shoot/ root/whole seedlings weight (fresh and dry), root, and shoot length and seedling height data. The performance of test lines/cultivars/controls are compared to the standards (controls), e.g. heat tolerant cv. N22, moderate tolerant cv. IR64 and/or sensitive cultivars IR 52, and/or WAB 56 104. Most of lines showed poor growth or death at temperatures higher than $45^{\circ} \mathrm{C}$ while some tolerant lines/cultivars showed better responses than sensitive. However tolerant lines showed slightly damaged compared to susceptible and highly susceptible lines, below scoring was used for classify genotypes/populations (Wei-hun et al. 2012). 

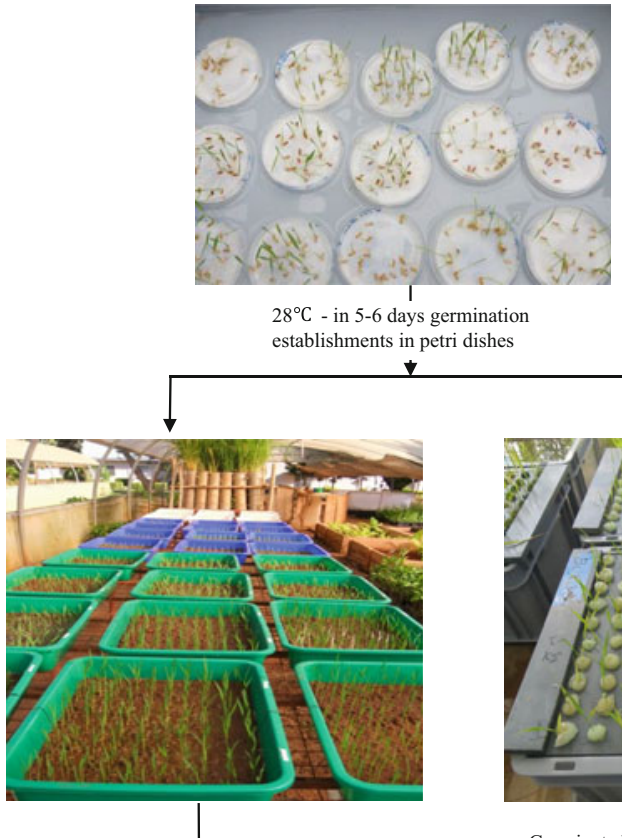

Germinated seedlings were

transplanted to trays in glasshouse

$28^{\circ} \mathrm{C}-15$ days
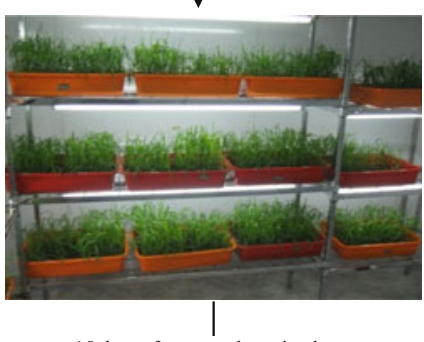

15 days after transplantation heat treatment $45^{\circ} \mathrm{C}-6$ hours

4-6 days in growth chamber
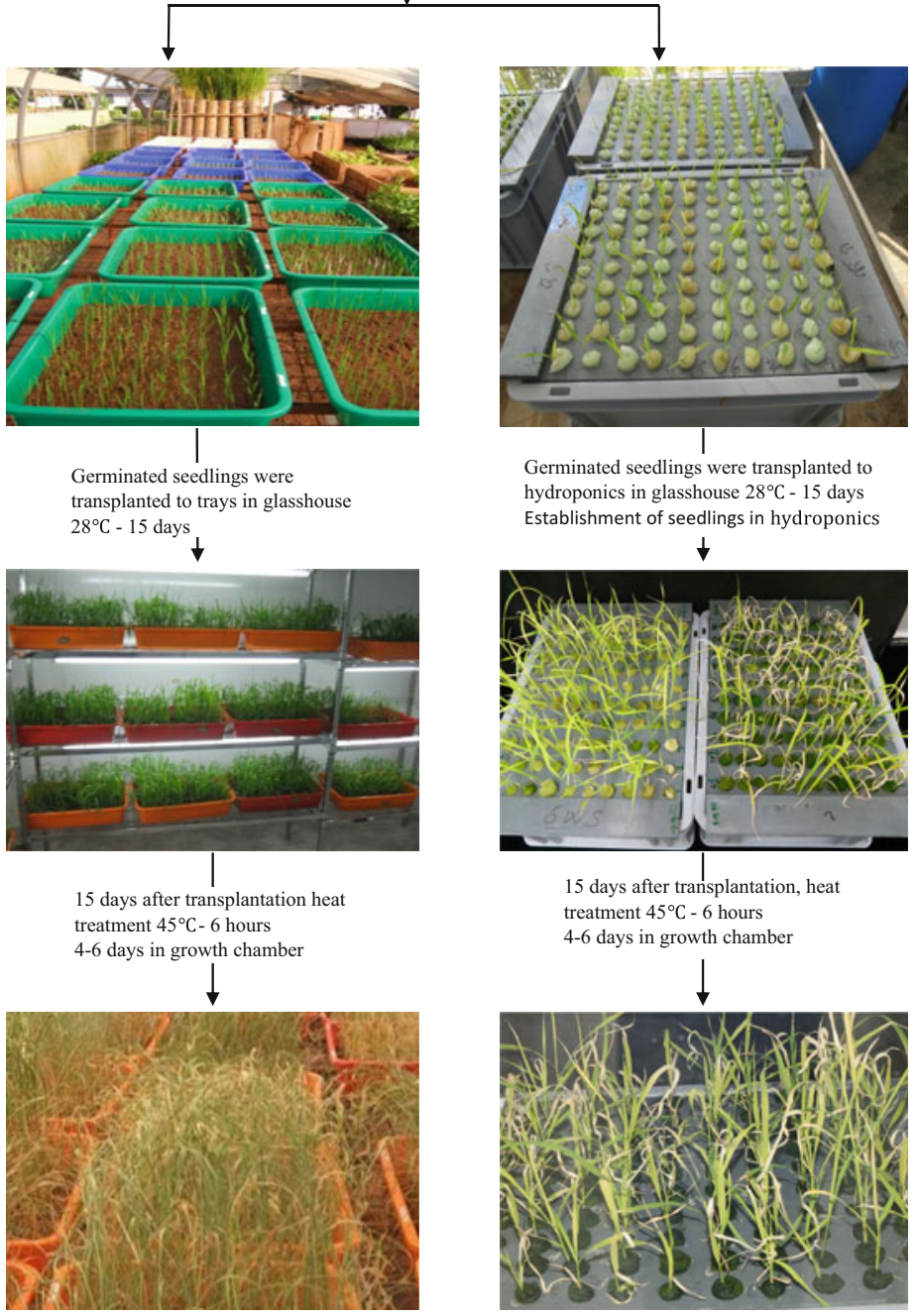

Germinated seedlings were transplanted to hydroponics in glasshouse $28^{\circ} \mathrm{C}-15$ days Establishment of seedlings in hydroponics

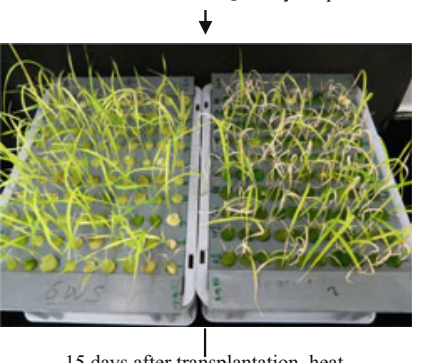

15 days after transplantation, heat treatment $45^{\circ} \mathrm{C}-6$ hours 4-6 days in growth chamber

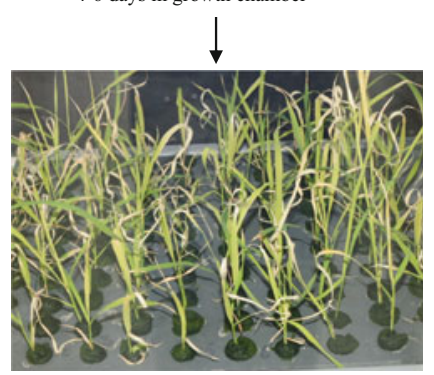

Assessment of germplasm according to the biomass of seedling *Same amount of seedling grown in glasshouse and evaluated

Fig. 2.5 Various steps of the protocol for screening rice seedlings for heat tolerance 
Table 2.1 Evaluation scores of seedlings for heat tolerance

\begin{tabular}{l|l|l}
\hline Score & Visual observation & Relative tolerance \\
\hline 1 & Nearly normal growth very rarely leaf rolling and leaf tips & Tolerant \\
\hline 2 & Most of leaves are rolled, yellowish and reduced leaves & Moderately tolerant \\
\hline 3 & Most of the leaves are dry and some of them dies & Susceptible \\
\hline 4 & Most of seedlings dying or already dead & Highly susceptible \\
\hline
\end{tabular}

\subsubsection{Heat Tolerance Index (HTI)}

After heat stress treatment, mutants were relocated to normal conditions with control plants. Two days after treatment data were recorded for shoot and root lengths, shoot and root fresh weights, shoot and root dry weights for stressed seedlings along with their respective controls grown under normal temperature.

A heat screening scale based on the response to stress was developed using parameters showing significant correlations with each other and with relative heat tolerance. Parameters were tested under control as well as heat stress and stress index was calculated for each parameter. The HTI is calculated by dividing the sum of individual scores for each parameter by the total score and multiplied by $100 \%$. The genotypes are graded from tolerant to sensitive.

Stress tolerance indices (STIs) for the recorded traits are calculated as follows (Fernandez 1992; Blum 1988):

$$
\mathrm{STI}=(\text { Value under stress } / \text { Value at control }) \times 100
$$

The genotypes were graded as tolerant, moderately tolerant, moderately sensitive and sensitive (Ashraf et al. 1999). Tables 2.2, 2.3, 2.4 and 2.5 summarize the data from one representative screening carried out on rice at the seedling stage in hydroponics and in pots and trays.

Table 2.2 Classification of heat tolerance in seedlings stage of various rice genotypes compared to medium heat tolerant cv. IR64 from NIAB Pakistan

\begin{tabular}{l|l|l}
\hline $\begin{array}{l}\text { More susceptible than } \\
\text { control cv. IR64 }\end{array}$ & Equivalent to control cv. IR64 & $\begin{array}{l}\text { More tolerant than control } \\
\text { cv. IR64 }\end{array}$ \\
\hline Tested 159 mutant lines & $\begin{array}{l}\text { HT-31, HT-51, HT-53, HT-81, } \\
\text { HT-104, HT-114, HT-138 }\end{array}$ & $\begin{array}{l}\text { HT-18, HT-39, HT-92, } \\
\text { HT-97, HT-98, HT-119 }\end{array}$ \\
\hline
\end{tabular}

Table 2.3 Classification of heat tolerance at the seedling stage of various rice genotypes compared to heat tolerant (HT) cv. N22 from FAO/IAEA PBGL, Seibersdorf, Austria

\begin{tabular}{l|l|l}
\hline More susceptible than control cv. N22 & $\begin{array}{l}\text { Equivalent to control } \\
\text { cv. N22 }\end{array}$ & $\begin{array}{l}\text { More tolerant than } \\
\text { control cv N22 }\end{array}$ \\
\hline LP-16, Super Basmati, IR 52, JC-1, JC-2, Saros & $\begin{array}{l}\text { 8552, LP 7, HT-53, } \\
\text { 5, Sahel 108, Sim 2 Sunade, Supa, G 619, WAB }\end{array}$ & $\begin{array}{l}\text { 8553, LP12, } \\
\text { HT-104, HT-132, }\end{array}$ \\
56 104, WAB 56 50, Pachaperumal, BG 300, BG & $\begin{array}{l}\text { HT-138, HT-119IR } \\
\text { 64, Sahel 317, HT-39, }\end{array}$ & \\
357, pokkali, bicol &
\end{tabular}


Table 2.4 Classification of heat tolerance at the seedling stage of various rice genotypes compared to heat tolerant (HT) cv. N22 from Jawaharlal Nehru University, India

\begin{tabular}{l|l|l}
\hline $\begin{array}{l}\text { More susceptible than control } \\
\text { cv. N22 }\end{array}$ & Equivalent to control cv. N22 & $\begin{array}{l}\text { More tolerant than control } \\
\text { cv. N22 }\end{array}$ \\
\hline Tested 108 mutant lines & $\begin{array}{l}\text { IR 64, D100/79 and } \\
\text { 200 mutant lines }\end{array}$ & $\begin{array}{l}\text { N22, D100/111 and D100/ } \\
96\end{array}$ \\
\hline
\end{tabular}

Table 2.5 Classification of heat tolerance at the seedling stage of 669 mutant lines compared to parental (wild type) and HT sensitive cv. WAB 56-104 from Sokoine University, UR Tanzania

\begin{tabular}{l|l|l}
\hline $\begin{array}{l}\text { More susceptible than control } \\
\text { WAB 56-104 and parent }\end{array}$ & $\begin{array}{l}\text { Equivalent to control cv WAB } \\
\text { 56-104 }\end{array}$ & $\begin{array}{l}\text { More tolerant than control cv } \\
\text { WAB 56-104 }\end{array}$ \\
\hline KR 28, KR 29, KR 30, CG14- & KR-9, KR-26, KR-39, KR-40, & KR 3, KR 10, KR 27, KR \\
20, CG14-52, CG14-53, CG14- \\
CG14-49, CG14-50, CG14-54, & 37, KR 38, CG14-5, CG14-6, \\
65, CG14-77, CG14-78, CG14- & CG14-51, CG14-60, CG14-66, & CG14-7, CG14-13, CG14-16, \\
80, CG14-79, CG14-81, & CG14-82, CG14-75, CG14-76, & CG14-21, CG14-22, CG14-59, \\
WAB56-104-39, WAB56-104- & WAB56-104-12, WAB56-104- & CG14-61, CG14-62, CG14-63, \\
40, WAB56-104-73, WAB56- & 46, WAB56-104-70, WAB56- & CG14-64, WAB56-104-9, \\
104-74, WAB56-104-132, & 104-62, WAB56-104-136, & WAB56-104-18, WAB56-104- \\
WAB56-104-133, WAB56- & WAB56-104-137, WAB56- & 36, WAB56-104-43, WAB56- \\
104-170, WAB56-104-171, & 104-119, WAB56-104-181, & 104-71, WAB56-104-76, \\
WAB56-50-59, WAB56-50- & WAB56-50-70, WAB56-50-90, & WAB56-104-118, WAB56- \\
60, WAB56-50-87, WAB56- & WAB56-50-91, WAB56-50- & $104-141$, WAB56-104-123, \\
50-88, WAB56-50-95, & 100, WAB56-50-107, WAB56- & WAB56-104-150, WAB56-50- \\
WAB56-50-96, WAB56-50- & 50-119, WAB56-50-139, & 48, WAB56-50-51, WAB56-50- \\
99, WAB56-50-124, WAB56- & WAB56-50-140, WAB56-50- & 56, WAB56-50-82, WAB56-50- \\
50-125, WAB56-50-143, & 146, WAB56-50-147 & 85, WAB56-50-97, WAB56-50- \\
WAB56-50-142, WAB56-50- & & 98, WAB56-50-123, WAB56- \\
133, WAB56-50-134 and & & $50-127$, WAB56-50-138, \\
WAB56-50-156 & & WAB56-50-141, WAB56-50- \\
& & 152, WAB56-50-135 \\
\hline
\end{tabular}

\subsubsection{Recovery Stage After Heat Treatment}

Heat tolerant lines that survive and recover after the heat stress at $45{ }^{\circ} \mathrm{C}$ may also be transplanted in hydroponics or soils. Selected seedlings can be grown on to maturity in hydroponic tanks filled with Yoshida solution changed every 2 weeks. Selected tolerant seedlings were gently teased out of the test tanks with care taken to keep roots intact. The base of the aerial part of each selected seedling is then wrapped in a sponge strip $(10 \times 2 \times 1 \mathrm{~cm})$ and the seedlings inserted into a recovery tank (Fig. 2.6a-c).

Heat tolerant lines may also be transplanted into 51 pots containing soil. The seedlings were maintained individually and single plants and allowed to form tillers and panicles. Preliminary data comparisons may be recorded on better lines with respect to: plant height, number of tillers, days to flowering, number of panicles, panicle length, sterility and fertility of panicle as well as yield data such as seed number, weight, and thousand grain weights for each individual plant per pot. Seeds from single plants are kept separately for multiplication in the next generation (Fig. 2.6d). 

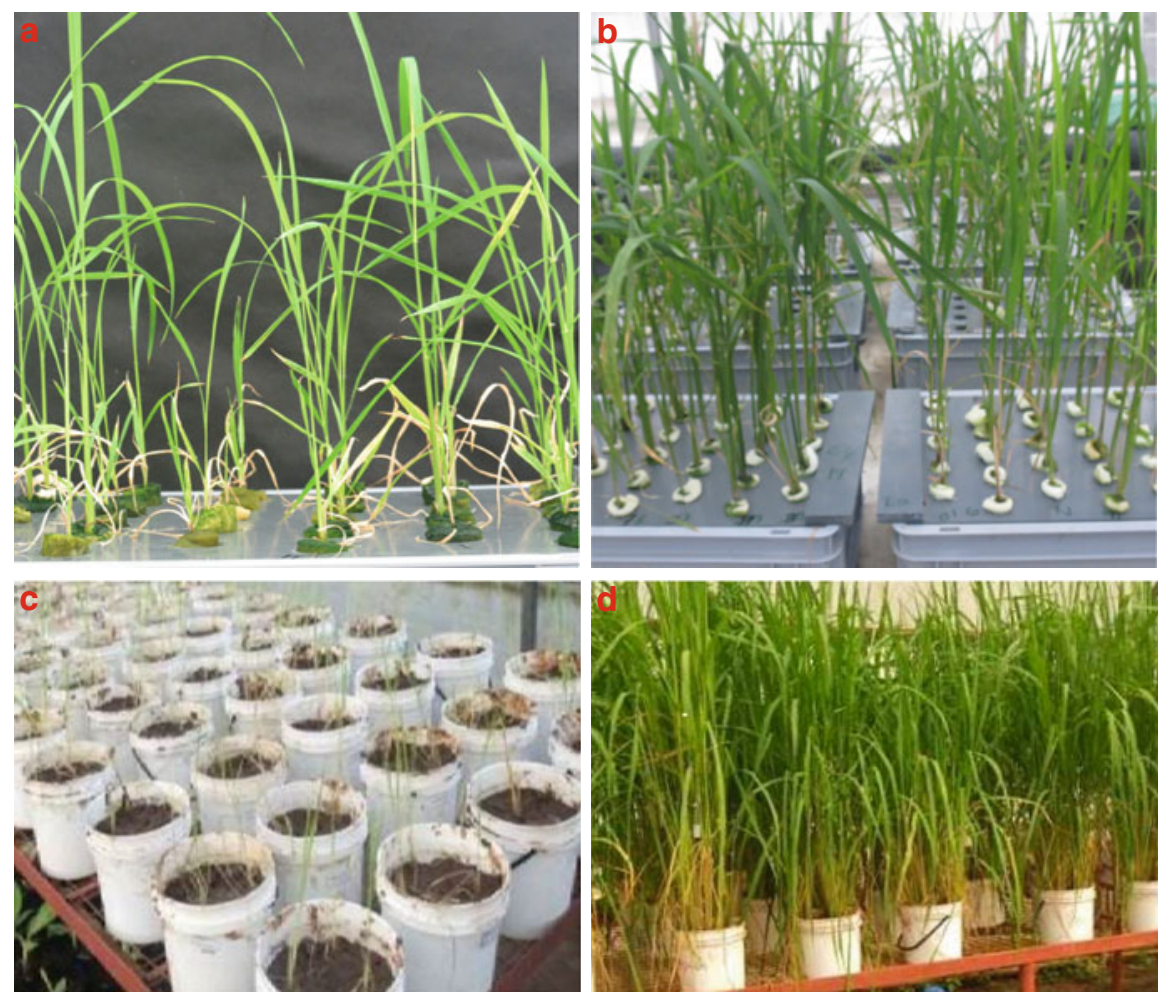

Fig. 2.6 Recovery of seedlings after heat treatment and transferto hydroponics or pots. Selected seedlings transferred to hydroponics and pots (a and c) and pots (c). These lines can be grown on until maturity (b and d) (Pictures from Joint FAO/IAEA Division PBGL Lab, Sokoine University in URT and Nuclear Institute for Agriculture and Biology (NIAB), Pakistan)

\subsection{Screening Protocol for Heat Tolerance in Rice at the Flowering Stage}

\subsubsection{Germination and Seedling Establishment in Hydroponics and Pots}

Uniform healthy seedlings (8-10 days old) are selected and grown individually in hydroponics (Fig. 2.7). The glasshouse set up for rice: $28 / 20^{\circ} \mathrm{C}$ day/night temperatures with $70 \%$ humidity and $16 \mathrm{~h}$ photoperiod. The hydroponics and pot culture set ups were the same as that described in Sects. 2.2 and 2.3. The glasshouse should be diseasefree and well-lit by natural and/or artificial lighting. 15-cm pots are recommended for single plants as these give enough seed yield and panicle number for line evaluation.

Every 2 weeks, the hydroponics solution needs to be changed. Hydroponics is also aerated by agitation twice in a week. Pots and boxes with soil culture were irrigated twice a week to maintain soil saturation. N, P, K fertiliser applications were done prior to transplanting and panicle emergence, which may be variable depending on the genotype. 

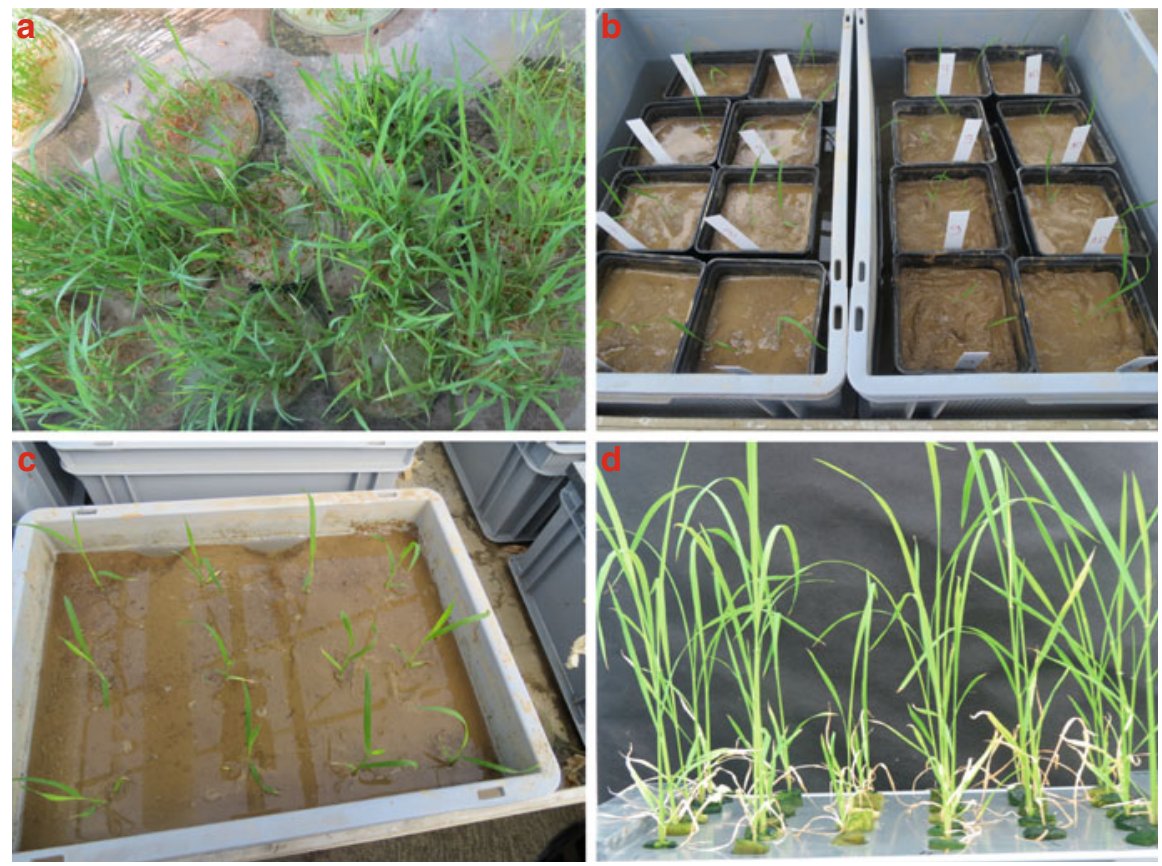

Fig. 2.7 (a) Pre-germinated rice seedlings in petri dishes, 8-10 day's old young seedlings transplanted to (b) pots and or (c) boxes (each box contains 9-10 plants). (d) Rice seedlings transplanted to hydroponics and grow on to the flowering stage (Pictures from Joint FAO/IAEA Division PBGL Lab)

Seedling establishment of control (standard) plant materials and test plant materials, care of seedlings in hydroponic, pots and boxes are same as described in Sects. 2.2 and 2.3 .

\subsubsection{Heat Treatment at the Flowering Stage}

Two treatments were applied to test genotypes (1) plants with no heat stress exposure (control) and (2) plants subjected to heat stress at flowering stage. Breeding lines and cultivars are used, with standards N22, IR64 as a heat tolerant/moderately tolerant standards; IR 52 and/or WAB 56100 were used as heat sensitive standards for comparative purposes. Plants were transferred to controlled environmental chambers/growth room conditions at the beginning of anthesis (first day) for heat stress treatments. Various heat stress treatments were investigated, all beginning at anthesis for various durations (2-6 $\mathrm{h}$ to $3-6$ days) and at various temperatures ranging from 35 to $39^{\circ} \mathrm{C}$. Here we describe the most effective treatment, at $39^{\circ} \mathrm{C}$ for 4 days, $6 \mathrm{~h}$ per day and $35^{\circ} \mathrm{C}, 6$ days, $6 \mathrm{~h}$ per day and $38^{\circ} \mathrm{C}, 5$ days and $4 \mathrm{~h}$ per day). 
First Treatment A set of 12 mutant lines (one plant grown per pot) were taken for testing high temperature tolerance. The main tiller of each plant was tagged before exposing plants to high temperature at flowering. On the appearance of the first flower on the main tiller plants were transferred to growth chambers at 07:00 $\mathrm{h}$ before the anthesis process starts. Environmental control chambers are set at $39{ }^{\circ} \mathrm{C}$ with 80\% relative humidity during the period of 6 hours 08:00-14:00 covering the anthesis period for each day. During anthesis, opened spikelets from four replicates (five spikelets from each plant) are marked with a permanent marker pen and moved to growth chamber for heat application.

Second Treatment Each genotype replicate consisted of ten pots with one to two plants per pot. Alternatively, 10 plants per genotype were grown up in soil filled boxes. Screens consisted of three replicates per genotype. Half (5) of the 10 pots or boxes were maintained in glasshouse conditions and served as controls. The other 5 were placed in a growth chamber and exposed to high temperatures at anthesis as described below. Illustration of heat stress treatments on rice at the flowering stage is given Fig. 2.8.

Heat stress treatment; At the beginning of anthesis, five pots per replicate of each genotype are transferred to growth chambers conditions in the morning 07:00 am with $80 \%$ humidity exposed to $35{ }^{\circ} \mathrm{C}$ for 6 days $6 \mathrm{~h}$, and $38{ }^{\circ} \mathrm{C} 5$ days $4 \mathrm{~h}$ respectively on consecutive days. The main tiller of each pot is tagged before exposing plants to high temperature at anthesis. At the first day of anthesis tagged plants were transferred to growth chambers set at $35-38{ }^{\circ} \mathrm{C}$ with $80 \%$ relative humidity as of from 7:00 AM every day. After each daily heat stress application, plants were returned to normal glasshouse conditions $\left(30 / 20{ }^{\circ} \mathrm{C}\right.$ day/night temperatures with $80 \%$ humidity). Plant phenotyping and heat stress treatment in growth chamber at flowering stage are shown in Fig. 2.9.
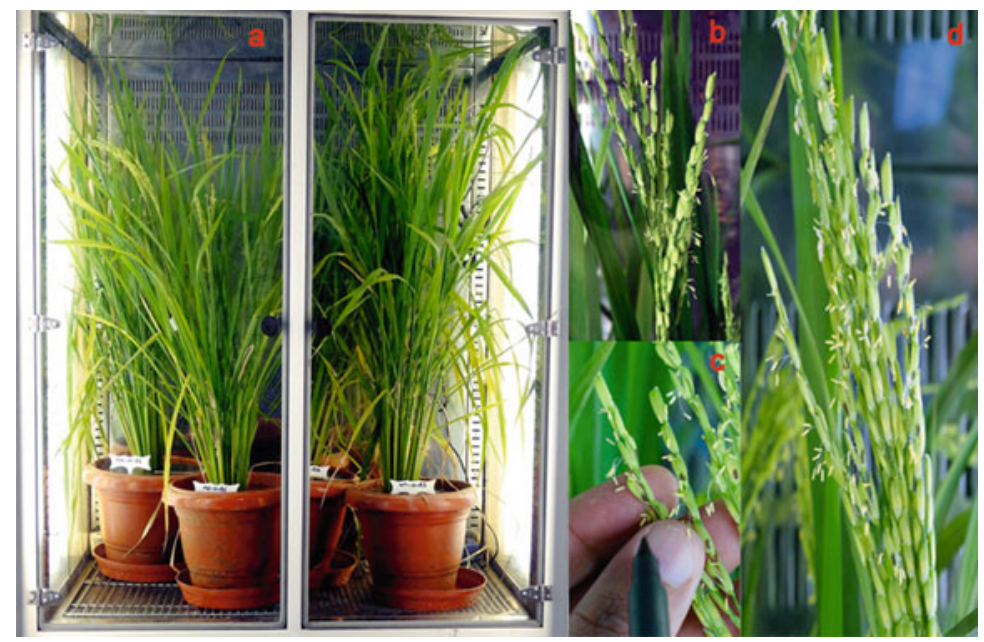

Fig. 2.8 Heat stress phenotyping in growth chambers at $35.0-39.0{ }^{\circ} \mathrm{C} / 28{ }^{\circ} \mathrm{C}$ (day/night) for $6-4 /$ $18-20 \mathrm{~h}$ for $6-4$ days $80 \%$ relative humidity (a, $\mathbf{c}$ and $\mathbf{~ d )}$ ). Heat stress application in growth chamber with pots, trays and hydroponics. (b) Enlarged view of panicles (Pictures from Jawaharlal Nehru University, India) 

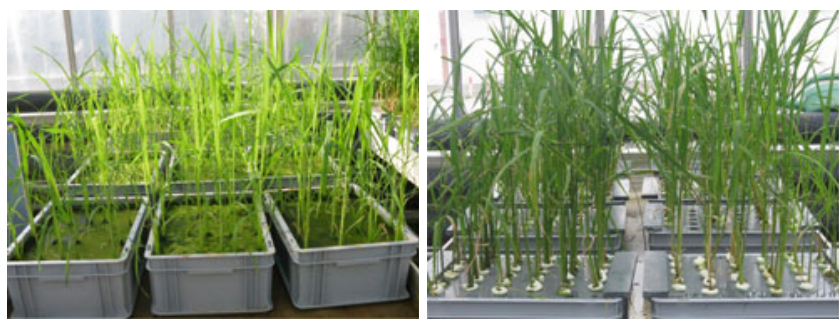

$28^{\circ} \mathrm{C}-6-8$ days, after germination seedlings transplanted to hydroponics or soil

I

Rice grown in glass house $28-30 / 20^{\circ} \mathrm{C}$ (day and night) till anthesis in trays/hydroponics

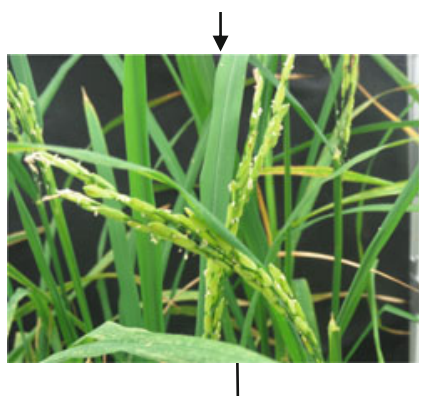

First day of anthesis main tillers are tagged plants transferred to growth chamber: $35^{0} \mathrm{C}, 6$ hours, 6 days or $38^{\circ} \mathrm{C}, 4$ hours, 5 days treatment or $39^{\circ} \mathrm{C}, 4$ and 4 days

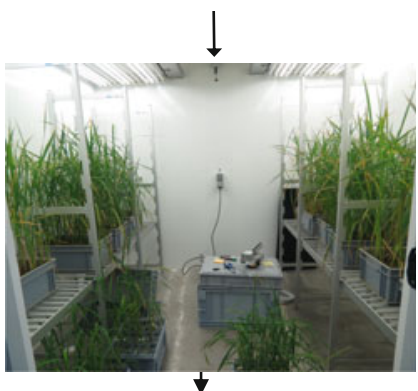

Assessment of germplasm according to the spikelet fertility

*Same number of plants grown in glasshouse and evaluated as controls

Fig. 2.9 Illustration of heat stress treatments on rice at the flowering stage 


\subsubsection{Screening for Heat Tolerance at the Flowering Stage}

Spikelet fertility at maturity was used to screen heat tolerance of tested genotypes. Plant height and tiller number per plant were recorded. Mature panicles were harvested from five remaining pots for grain yield per plant recording, grain quality traits and calculating spikelet fertility (by counting empty and grain-filled spikelets). Main tillers were tagged and after harvest panicles were manually threshed, numbers of filled and unfilled grains per panicle were recorded. Each floret can be pressed between forefinger and thumb to determine if the grain is filled or not. Both partially and fully filled spikelets were categorized as filled spikelets.

Spikelet fertility is calculated as below;

$$
\text { Spikelet fertility }=\frac{\text { Number of filled grains }}{\text { Total number of reproductive sites (floret) }} \times 100
$$

Spikelet fertility was calculated in control and high temperature stress conditions. The negative effect ( $\%$ decrease from control) was determined per genotype.

Reduced fertility of more than $65 \%$ is classed as highly susceptible, between 25-65\% classed as intermediate (moderately susceptible) and less than $25 \%$ is classified as heat tolerant. This quantification has been adapted from species, ecotype and cultivar differences in spikelet fertility and harvest index of rice in response to high temperature stress (Prasad et al. 2006; Rang et al. 2011) (Tables 2.6, 2.7 and 2.8).

Table 2.6 Classification of heat tolerance at the seedling stage of various rice genotypes compared to the heat tolerant $(\mathrm{HT}) \mathrm{cv}$. N 22 from FAO/IAEA PBG Seibersdorf, Austria, (treatment $35^{\circ} \mathrm{C}$ for 6 days, $6 \mathrm{~h}$ per days)

\begin{tabular}{l|l|l}
\hline $\begin{array}{l}\text { More susceptible than } \\
\text { control cv. N22 } \\
\text { Susceptible }>65 \% \text { sterility }\end{array}$ & $\begin{array}{l}\text { Equivalent to control cv. N22 } \\
\text { Moderate tolerance: sterility } \\
\text { from 25-65\% }\end{array}$ & $\begin{array}{l}\text { More tolerant than control } \\
\text { cv. N22 } \\
\text { Tolerant lines: sterility }<25 \%\end{array}$ \\
\hline $\begin{array}{l}\text { LP-16, WAB 56 104, WAB } \\
56-50\end{array}$ & LP 7, IR 64, HT-39, HT-119, & $\begin{array}{l}8553, \text { LP12, HT-97, II You } \\
838, \text { N 22, HT 74 }\end{array}$ \\
\hline
\end{tabular}

Table 2.7 Classification of heat tolerance at the flowering stage of various rice genotypes from Jawaharlal Nehru University, India compared to the heat tolerant (HT) cv. N 22 (treatment $39^{\circ} \mathrm{C}$ for 4 days, $4 \mathrm{~h}$ per day)

\begin{tabular}{l|l|l}
\hline $\begin{array}{l}\text { More susceptible than control } \\
\text { cv. N22 }\end{array}$ & $\begin{array}{l}\text { Equivalent to control cv. N22. } \\
\text { Moderate tolerant sterility from }\end{array}$ & $\begin{array}{l}\text { More tolerant than control } \\
\text { cv. N22 } \\
\text { Tolerant line } \\
\text { sterility }<25 \%\end{array}$ \\
\hline $\begin{array}{l}\text { Susceptible }>65 \% \text { sterility } \\
\text { Tested } 90 \text { lines are susceptible } \\
\text { than IR 64 }\end{array}$ & IR 64 and D100/79 & $\begin{array}{l}\text { N22, D100/111 and D100/ } \\
96\end{array}$ \\
\hline
\end{tabular}


Table 2.8 Classification of heat tolerance at the flowering stage of mutant lines compare to parental (wild type) genotypes and HT sensitive cv. WAB 56-104 and parental lines from Sokoine University, UR Tanzania (treatment $38^{\circ} \mathrm{C}$ for 5 days, $4 \mathrm{~h}$ per days)

\begin{tabular}{l|l|l}
\hline $\begin{array}{l}\text { More susceptible than control } \\
\text { cv.WAB 56 }\end{array}$ & $\begin{array}{l}\text { Equivalent to control cv. WAB } \\
56-104 . \\
\text { Moderate tolerant sterility from } \\
\text { Susceptible }>\text { 65\% sterility }\end{array}$ & $\begin{array}{l}\text { More tolerant than to control } \\
\text { cv WAB 56-104 } \\
\text { Tolerant lines sterility < 25\% }\end{array}$ \\
\hline KR-3; KR-38; CG14-59; & KR-27; CG14-5; CG14-6; & KR-37; CG14-13; CG14-16; \\
CG14-63; CG14-64; WAB56- & CG14-7; CG14-61; CG14-62; & CG14-21; CG14-22; WAB56- \\
104-18; WAB56-104-118; & WAB56-104-141; WAB56-50- & 104-9; WAB56-104-36; \\
WAB56-104-123; WAB56-50- & 51; WAB56-50-97; WAB56- & WAB56-104-43; WAB56- \\
85; WAB56-50-98; WAB56- & 50-123; WAB56-50-127; & 104-71; WAB56-104-76; \\
50-141; WAB56-50-152 & WAB56-50-135 & WAB56-104-150; WAB56- \\
& & 50-48; WAB56-50-96; \\
& & WAB56-50-82; WAB56-50- \\
& & 138 \\
\hline
\end{tabular}

Open Access This chapter is licensed under the terms of the Creative Commons Attribution 3.0 IGO license (https://creativecommons.org/licenses/by/3.0/igo/), which permits use, sharing, adaptation, distribution and reproduction in any medium or format, as long as you give appropriate credit to the International Atomic Energy Agency (IAEA), provide a link to the Creative Commons license and indicate if changes were made.

Any dispute related to the use of the works of the IAEA that cannot be settled amicably shall be submitted to arbitration pursuant to the UNCITRAL rules. The use of the IAEA's name for any purpose other than for attribution, and the use of the IAEA's logo, shall be subject to a separate written license agreement between the IAEA and the user and is not authorized as part of this CC-IGO license. Note that the link provided above includes additional terms and conditions of the license.

The images or other third party material in this chapter are included in the chapter's Creative Commons license, unless indicated otherwise in a credit line to the material. If material is not included in the chapter's Creative Commons license and your intended use is not permitted by statutory regulation or exceeds the permitted use, you will need to obtain permission directly from the copyright holder.

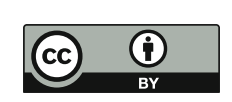

